TRANSACTIONS OF THE

AMERICAN MATHEMATICAL SOCIETY

Volume 357, Number 9 , Pages $3655-3672$

S $0002-9947(05) 03758-X$

Article electronically published on January 21, 2005

\title{
ASYMPTOTIC BEHAVIOUR OF ARITHMETICALLY COHEN-MACAULAY BLOW-UPS
}

\author{
HUY TÀI HÀ AND NGÔ VIÊT TRUNG
}

\begin{abstract}
This paper addresses problems on arithmetic Macaulayfications of projective schemes. We give a surprising complete answer to a question poised by Cutkosky and Herzog. Let $Y$ be the blow-up of a projective scheme $X=\operatorname{Proj} R$ along the ideal sheaf of $I \subset R$. It is known that there are embeddings $Y \cong \operatorname{Proj} k\left[\left(I^{e}\right)_{c}\right]$ for $c \geq d(I) e+1$, where $d(I)$ denotes the maximal generating degree of $I$, and that there exists a Cohen-Macaulay ring of the form $k\left[\left(I^{e}\right)_{c}\right]$ (which gives an arithmetic Macaulayfication of $X$ ) if and only if $H^{0}\left(Y, \mathcal{O}_{Y}\right)=k, H^{i}\left(Y, \mathcal{O}_{Y}\right)=0$ for $i=1, \ldots, \operatorname{dim} Y-1$, and $Y$ is equidimensional and Cohen-Macaulay. We show that under these conditions, there are well-determined invariants $\varepsilon$ and $e_{0}$ such that $k\left[\left(I^{e}\right)_{c}\right]$ is Cohen-Macaulay for all $c>d(I) e+\varepsilon$ and $e>e_{0}$, and that these bounds are the best possible. We also investigate the existence of a Cohen-Macaulay Rees algebra of the form $R\left[\left(I^{e}\right)_{c} t\right]$. If $R$ has negative $a^{*}$-invariant, we prove that such a CohenMacaulay Rees algebra exists if and only if $\pi_{*} \mathcal{O}_{Y}=\mathcal{O}_{X}, R^{i} \pi_{*} \mathcal{O}_{Y}=0$ for $i>0$, and $Y$ is equidimensional and Cohen-Macaulay. Moreover, these conditions imply the Cohen-Macaulayness of $R\left[\left(I^{e}\right)_{c} t\right]$ for all $c>d(I) e+\varepsilon$ and $e>e_{0}$.
\end{abstract}

\section{INTRODUCTION}

Let $X$ be a projective scheme over a field $k$. An arithmetic Macaulayfication of $X$ is a proper birational morphism $\pi: Y \rightarrow X$ such that $Y$ has an arithmetically Cohen-Macaulay embedding, i.e. there exists a Cohen-Macaulay standard graded $k$-algebra $A$ such that $Y \cong \operatorname{Proj} A$. Inspired by the problem of resolution of singularities, it was asked when $X$ has an arithmetic Macaulayfication. The local version of this problem (arithmetic Macaulayfication of local rings) has been extensively studied in the literature and recently solved by Kawasaki [22. An important aspect of the global problem is to determine, given a proper birational morphism $Y \rightarrow X$, if $Y$ has an arithmetically Cohen-Macaulay embedding, and if it does, which embeddings of $Y$ are arithmetically Cohen-Macaulay.

Let $R$ be a standard graded $k$-algebra and let $I \subset R$ be a homogeneous ideal such that $X=\operatorname{Proj} R$ and $Y$ is the blow-up of $X$ along the ideal sheaf of $I$. It was observed by Cutkosky and Herzog [9] that $Y \cong \operatorname{Proj} k\left[\left(I^{e}\right)_{c}\right]$ for $c \geq d(I) e+1$,

Received by the editors January 10, 2004.

2000 Mathematics Subject Classification. Primary 14M05, 13H10, 13A30, 14E25.

Key words and phrases. Blow-up, Rees algebra, Cohen-Macaulay, projective embedding.

The second author was partially supported by the National Basic Research Program of Vietnam.

(C)2005 American Mathematical Society Reverts to public domain 28 years from publication 
where $\left(I^{e}\right)_{c}$ denotes the vector space of forms of degree $c$ of the ideal power $I^{e}$ and $d(I)$ is the maximal degree of the elements of a homogeneous basis of $I$. In other words, $Y$ can be embedded into a projective space by the complete linear system $\left|c E_{0}-e E\right|$, where $E$ denotes the exceptional divisor and $E_{0}$ is the pull-back of a general hyperplane in $X$. By [26] we know that there exists a Cohen-Macaulay ring $k\left[\left(I^{e}\right)_{c}\right]$ for $c \geq d(I) e+1$ if and only if $Y$ satisfies the following conditions:

- $Y$ is equidimensional and Cohen-Macaulay,

- $H^{0}\left(Y, \mathcal{O}_{Y}\right)=k$ and $H^{i}\left(Y, \mathcal{O}_{Y}\right)=0$ for $i=1, \ldots, \operatorname{dim} Y-1$.

In the first part of this paper, we study the problem of which values of $c$ and $e$ is $k\left[\left(I^{e}\right)_{c}\right]$ a Cohen-Macaulay ring. This problem originated from a beautiful result of Geramita, Gimigliano and Pitteloud [13] which shows that if $I$ is the defining ideal of a set of fat points in a projective space over a field of characteristic zero, then $k\left[I_{c}\right]$ is a Cohen-Macaulay ring for all $c \geq \operatorname{reg}(I)$, where $\operatorname{reg}(I)$ is the CastelnuovoMumford regularity of $I$. This result initiated the study on the Cohen-Macaulayness of algebras of the form $k\left[\left(I^{e}\right)_{c}\right]$ first in 7 and then in [9, 25, 26, 16. In particular, Cutkosky and Herzog [9] showed that if $I$ is a locally complete intersection ideal, then there exists a constant $\delta$ such that $k\left[\left(I^{e}\right)_{c}\right]$ is Cohen-Macaulay for $c \geq \delta e$. They raised the question of when there is a linear bound on $c$ and $e$ ensuring that $k\left[\left(I^{e}\right)_{c}\right]$ is a Cohen-Macaulay ring.

Our results will give a complete answer to this question. We show that if the above two conditions are satisfied, then there exist well-determined invariants $\varepsilon$ and $e_{0}$ such that $k\left[\left(I^{e}\right)_{c}\right]$ is a Cohen-Macaulay ring for all $c>d(I) e+\varepsilon$ and $e>e_{0}$ (Theorem [2.2). The invariant $e_{0}$ is a projective version of the $a^{*}$-invariant, which is the largest non-vanishing degree of the graded local cohomology modules 29 , 32. The invariant $\varepsilon$ comes from the asymptotic linearity of the CastelnuovoMumford regularity of powers of ideals (31, 10, 23, 34]). We will see that the bounds $c>d(I) e+\varepsilon$ and $e>e_{0}$ are the best possible (Theorem 2.3 and Example 2.5). The existence of linear bounds on $c$ and $e$ is not hard to prove. The novelty we claim here is that an explicit description for the best possible bounds is obtained. Moreover, if the Rees algebra $R[I t]$ is locally Cohen-Macaulay on $X$, then $e_{0}=0$ and we can replace the second condition by the weaker condition that $H^{0}\left(X, \mathcal{O}_{X}\right)=k$ and $H^{i}\left(X, \mathcal{O}_{X}\right)=0$ for $i=1, \ldots, \operatorname{dim} X-1$ (Theorem 2.4). These results strengthen and unify all previously-known results on the Cohen-Macaulayness of $k\left[\left(I^{e}\right)_{c}\right]$ which were obtained by different methods.

In the second part of this paper, we investigate the more difficult question of when $Y$ is an arithmetically Cohen-Macaulay blow-up of $X$; that is, when there exists a standard graded $k$-algebra $R$ and an ideal $J \subset R$, such that $X=\operatorname{Proj} R$, $Y$ is the blow-up of $X$ along the ideal sheaf of $J$, and $R[J t]$ is a Cohen-Macaulay ring. Given $R$ and $I$, we will concentrate on ideals $J \subseteq I$ which are generated by the elements of $\left(I^{e}\right)_{c}$. It is obvious that $I^{e}$ and $J$ define the same ideal sheaf for $c \geq d(I) e$. Rees algebras of the form $R\left[I_{c} t\right](e=1)$ have been studied first for the defining ideal of a set of points in [15] and then for locally complete intersection ideals in [8], where it was shown that there exists a constant $\lambda$ such that $R\left[I_{c} t\right]$ is a Cohen-Macaulay ring for $c \geq \lambda$. This leads to the problem of whether there is a constant $\delta$ such that the Rees algebra $R\left[\left(I^{e}\right)_{c} t\right]$ is a Cohen-Macaulay ring for $c \geq \delta e$.

If $a^{*}(R)<0$ (e.g. if $R$ is a polynomial ring) we solve this problem by showing that there exists a Cohen-Macaulay ring $R\left[\left(I^{e}\right)_{c} t\right]$ with $c \geq d(I) e$ if and only if the 
following conditions are satisfied:

- $Y$ is equidimensional and Cohen-Macaulay,

- $\pi_{*} \mathcal{O}_{Y}=\mathcal{O}_{X}, R^{i} \pi_{*} \mathcal{O}_{Y}=0$ for $i>0$.

In particular, these conditions imply that $R\left[\left(I^{e}\right)_{c} t\right]$ is a Cohen-Macaulay ring for all $c>d(I) e+\varepsilon$ and $e>e_{0}$ (Theorem 3.4). From this it follows that there exists a Cohen-Macaulay algebra of the form $R\left[I_{c} t\right]$ with $c \geq d(I)$ if and only if $R[I t]$ is locally Cohen-Macaulay on $X$ and that $e_{0}=0$ in this case (Corollary 3.8). We would like to point out that this phenomenon does not hold in general. In fact, there exist examples with $a^{*}(R) \geq 0$ such that $R\left[\left(I^{e}\right)_{d(I) e} t\right]$ is a Cohen-Macaulay ring, whereas $R\left[\left(I^{e}\right)_{c} t\right]$ is not a Cohen-Macaulay ring for any $c>d(I) e$ (Example 3.5). Using the above result we obtain several new classes of Cohen-Macaulay Rees algebras. Furthermore, we show that if $H^{0}\left(X, \mathcal{O}_{X}\right)=k$ and $H^{i}\left(X, \mathcal{O}_{X}\right)=0$ for $i>0$, then $Y$ is an arithmetically Cohen-Macaulay blow-up of $X$ if and only if $Y$ is locally arithmetical Cohen-Macaulay on $X$ (Theorem 3.12).

Our approach is based on the facts that the Rees algebra $S=R[I t]$ has a natural bi-gradation and that $k\left[\left(I^{e}\right)_{c}\right]$ can be viewed as a diagonal subalgebra of $S$ [7]. As a consequence, the Cohen-Macaulayness of $k\left[\left(I^{e}\right)_{c}\right]$ can be characterized by means of the sheaf cohomology $H^{i}\left(Y, \mathcal{O}_{Y}(m, n)\right)$. Using Leray spectral sequence and SerreGrothendieck correspondence, we may pass from this sheaf cohomology to the local cohomology of $I^{n}$ and of $\omega_{n}$, where $\omega_{S}=\bigoplus_{n \in \mathbb{Z}} \omega_{n}$ denotes the graded canonical module of $S$. It was shown recently that there are linear bounds for the vanishing of the local cohomology of $I^{n}$ and $\omega_{n}([31,10,23,34)$. It turns out that these linear bounds yield a linear bound on $c$ and $e$ such that $k\left[\left(I^{e}\right)_{c}\right]$ is a Cohen-Macaulay ring. The Cohen-Macaulayness of the Rees algebra $R\left[\left(I^{e}\right)_{c} t\right]$ can be studied similarly by using a recent result of Hyry [19] which characterizes the Cohen-Macaulayness of a standard bi-graded algebra by means of sheaf cohomology.

The paper is organized as follows. In Section 1, we introduce the notion of a projective $a^{*}$-invariant which governs how sheaf cohomology behaves through blowing up morphisms. The material in this section is interesting on its own right. In Section 2, we study the Cohen-Macaulayness of rings of the form $k\left[\left(I^{e}\right)_{c}\right]$ which correspond to projective embeddings of $Y$. The last section of the paper deals with the problem of when $Y$ is an arithmetically Cohen-Macaulay blow-up of $X$.

For unexplained notation and facts we refer the reader to the books [4, 5, 17].

\section{1. $a^{*}$-INVARIANTS}

Let $R$ be an arbitrary commutative noetherian ring. Let $S=\bigoplus_{n>0} S_{n}$ be a finitely generated graded algebra over $R$. We shall always use $S_{+}=\bigoplus_{n>0} S_{n}$ to denote the ideal generated by the homogeneous elements of positive degrees of $S$. Given any finitely generated graded $S$-module $F$, the local cohomology module $H_{S_{+}}^{i}(F)$ is also a graded $S$-module. It is well known that $H_{S_{+}}^{i}(F)_{n}=0$ for $n \gg 0$, $i \geq 0$. Put

$$
a_{i}(F)=\left\{\begin{array}{lll}
-\infty & \text { if } & H_{S_{+}}^{i}(F)=0, \\
\max \left\{n \mid H_{S_{+}}^{i}(F)_{n} \neq 0\right\} & \text { if } & H_{S_{+}}^{i}(F) \neq 0 .
\end{array}\right.
$$

Note that $a(F):=a_{\operatorname{dim} F}(F)$ is called the $a$-invariant of $F$ if $S$ is a standard graded algebra over a field. The $a^{*}$-invariant of $F$ is defined to be

$$
a^{*}(F):=\max \left\{a_{i}(F) \mid i \geq 0\right\} .
$$


This invariant was introduced in [32] and [29] in order to control the vanishing of graded local cohomology modules with different supports. It is closely related to the Castelnuovo-Mumford regularity via the equality

$$
\operatorname{reg}(F)=\max \left\{a_{i}(F)+i \mid i \geq 0\right\} .
$$

Here we are interested in the case when $R$ is a standard graded algebra over a field $k$ and $S=R[I t]$ is the Rees algebra of a homogeneous ideal $I \subset R$ with ht $I \geq 1$. This Rees algebra has a natural grading with $S_{n}=I^{n} t^{n}$. Let $\omega_{S}=\bigoplus_{n \in \mathbb{Z}} \omega_{n}$ denote the canonical graded module of $S$.

Lemma 1.1. Let $S=R[I t]$ be as above. If $S$ is a Cohen-Macaulay ring, then $a^{*}(S)=-1$ and $a^{*}\left(\omega_{S}\right)=0$.

Proof. It is well known that $\operatorname{dim} S=\operatorname{dim} R+1$. Since $S / S_{+}=R$, we have ht $S_{+}=$ $\operatorname{dim} S-\operatorname{dim} R=1$. This implies grade $S_{+}=1$. Hence $a^{*}(S) \geq-1$ by 32 , Corollary 2.3]. On the other hand, the Cohen-Macaulayness of $S$ implies $H_{M}^{i}(S)=0$ for $i<\operatorname{dim} S$, where $M$ denotes the maximal graded ideal of $S$. By [33, Corollary 3.2] we always have $H_{M}^{\operatorname{dim} S}(S)_{n}=0$ for $n \geq 0$. Hence $H_{M}^{i}(S)_{n}=0$ for all $n \geq 0$ and $i \geq 0$. By [19] Lemma 2.3] (or [32, Corollary 2.8]), this implies $H_{S_{+}}^{i}(S)_{n}=0$ for all $n \geq 0$ and $i \geq 0$. Therefore, $a^{*}(S)=-1$.

Since $\omega_{S}$ is a Cohen-Macaulay module with $\operatorname{Hom}_{S}\left(\omega_{S}, \omega_{S}\right) \cong S[2$ Proposition 2], we also have $H_{M}^{i}\left(\omega_{S}\right)=0$ for $i<\operatorname{dim} S$ and, by local duality,

$$
H_{M}^{\operatorname{dim} S}\left(\omega_{S}\right)_{n} \cong \operatorname{Hom}_{S}\left(\omega_{S}, \omega_{S}\right)_{-n} \cong S_{-n} .
$$

Since $S_{0}=R \neq 0$ and $S_{-n}=0$ for $n>0$, we can conclude that $a_{X}^{*}\left(\omega_{S}\right)=0$.

Let $X=\operatorname{Proj} R$. For each $\mathfrak{p} \in X$, the homogeneous localization $F_{(\mathfrak{p})}$ is a finitely generated graded module over $S_{(\mathfrak{p})}$. Hence, we can define the projective $a^{*}$-invariant

$$
a_{X}^{*}(F):=\max \left\{a^{*}\left(F_{(\mathfrak{p})}\right) \mid \mathfrak{p} \in X\right\} .
$$

Note that $H_{S_{(\mathfrak{p})+}}^{i}\left(F_{(\mathfrak{p})}\right)=H_{S_{+}}^{i}(F)_{(\mathfrak{p})}$ (cf. 29, Remark 2.2]). Then we always have $a_{X}^{*}(F) \leq a^{*}(F)$. Hence $a_{X}^{*}(F)$ is a finite number. Since $a_{X}^{*}(F)$ is determined by the local structure of $F$ on $X$, it can easily be estimated in certain situations. As a demonstration, we show how to estimate $a_{X}^{*}(F)$ in the following case which will play an important role in our further investigation.

We say that $S$ is locally Cohen-Macaulay on $X$ if $S_{(\mathfrak{p})}$ is a Cohen-Macaulay ring for every $\mathfrak{p} \in X$. This condition holds if, for instance, $X$ is locally Cohen-Macaulay and $\mathcal{I}$ is locally a complete intersection.

Proposition 1.2. Let $X=\operatorname{Proj} R$ and $S=R[I t]$ be as above. Then $a_{X}^{*}(S) \geq-1$ and $a_{X}^{*}\left(\omega_{S}\right) \geq 0$. Equalities hold if $S$ is locally Cohen-Macaulay on $X$.

Proof. Let $\mathfrak{p}$ be a minimal prime ideal in $X$. Then $R_{(\mathfrak{p})}$ is an artinian ring. Since $\mathfrak{p} \nsupseteq I$, we have $I_{(\mathfrak{p})}=R_{(\mathfrak{p})}$. Hence $S_{(\mathfrak{p})}=R_{(\mathfrak{p})}[t]$ is a Cohen-Macaulay ring. By Lemma 1.1, this implies $a^{*}\left(S_{(\mathfrak{p})}\right)=-1$ and $a^{*}\left(\omega_{S_{(\mathfrak{p})}}\right)=0$. Hence $a_{X}^{*}(S) \geq-1$ and $a_{X}^{*}\left(\omega_{S}\right) \geq 0$. This proves the first statement. The second statement is an immediate consequence of Lemma 1.1 .

Beside the natural $\mathbb{N}$-graded structure given by the degrees of $t$, the Rees algebra $S=R[I t]$ also has a natural bi-gradation with

$$
S_{(m, n)}=\left(I^{n}\right)_{m} t^{n}
$$


for $(m, n) \in \mathbb{N}^{2}$. Let $Y$ be the blow-up of $X$ along the ideal sheaf of $I$. Then $Y=\operatorname{Proj} S$ with respect to this bi-gradation. If $F=\bigoplus_{(m, n) \in \mathbb{Z}^{2}} F_{(m, n)}$ is a finitely generated bi-graded $S$-module, then $F$ is also an $\mathbb{Z}$-graded $S$-module with $F_{n}=$ $\bigoplus_{m \in \mathbb{Z}} F_{(m, n)}$. Let $\widetilde{F}$ denote the sheaf associated to $F$ on $Y$. We write $\tilde{F}(n)$ and $\tilde{F}(m, n)$ to denote the twisted $\mathcal{O}_{Y}$-modules with respect to the $\mathbb{N}$-gradation and the $\mathbb{N}^{2}$-gradation of $S$. Moreover, we denote by $\widetilde{F_{n}}$ the sheafification of $F_{n}$ on $X$.

It turns out that $a_{X}^{*}(F)$ is a measure for when we can pass from the sheaf cohomology of $\widetilde{F}(m, n)$ on $Y$ to that of $\widetilde{F_{n}}(m)$ on $X$.

Proposition 1.3. Let $F$ be a finitely generated bi-graded $S$-module. For $n>a_{X}^{*}(F)$ we have

(i) $\pi_{*}(\widetilde{F}(n))=\widetilde{F_{n}}$ and $R^{i} \pi_{*}(\widetilde{F}(n))=0$ for $i>0$,

(ii) $H^{i}(Y, \widetilde{F}(m, n)) \cong H^{i}\left(X, \widetilde{F_{n}}(m)\right)$ for all $m \in \mathbb{Z}$ and $i \geq 0$.

Proof. Since (i) is a local statement, we only need to show that it holds locally. Let $\mathfrak{p}$ be a closed point of $X$, and consider the restriction $\pi_{\mathfrak{p}}$ of $\pi$ over an affine open neighborhood $\operatorname{Spec} \mathcal{O}_{X, \mathfrak{p}}$ of $\mathfrak{p}$

$$
\pi_{\mathfrak{p}}: Y_{\mathfrak{p}}=Y \times_{X} \operatorname{Spec} \mathcal{O}_{X, \mathfrak{p}} \rightarrow \operatorname{Spec} \mathcal{O}_{X, \mathfrak{p}}
$$

We have $\left.\tilde{F}\right|_{Y_{\mathfrak{p}}}=\widetilde{F_{(\mathfrak{p})}}$, where $\widetilde{F_{(\mathfrak{p})}}$ is the sheaf associated to $F_{(\mathfrak{p})}$ on $Y_{\mathfrak{p}}$. Thus,

$$
\left.R^{i} \pi_{*}(\tilde{F}(n))\right|_{\operatorname{Spec} \mathcal{O}_{X, \mathfrak{p}}}=R^{i} \pi_{\mathfrak{p}_{*}}\left(\widetilde{F_{(\mathfrak{p})}}(n)\right)=H^{i}\left(Y_{\mathfrak{p}}, \widetilde{F_{(\mathfrak{p})}}(n)\right)^{\sim}
$$

On the other hand, we know by the Serre-Grothendieck correspondence that there are the exact sequence

$$
0 \rightarrow H_{S_{(\mathfrak{p})+}}^{0}\left(F_{(\mathfrak{p})}\right)_{n} \rightarrow\left(F_{(\mathfrak{p})}\right)_{n} \rightarrow H^{0}\left(Y_{\mathfrak{p}}, \widetilde{F_{(\mathfrak{p})}}(n)\right) \rightarrow H_{S_{(\mathfrak{p})+}}^{1}\left(F_{(\mathfrak{p})}\right)_{n} \rightarrow 0
$$

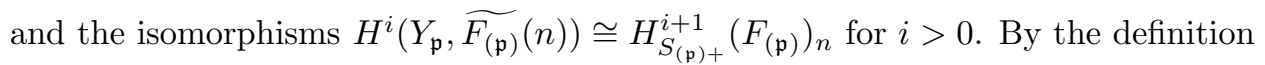
of $a_{X}^{*}(F)$, we know that $H_{S_{(\mathfrak{p})+}^{i}}^{i}\left(F_{(\mathfrak{p})}\right)_{n}$ for $n>a_{X}^{*}(F), i>0$. Thus,

$$
\left.R^{i} \pi_{*}(\tilde{F}(n))\right|_{\operatorname{Spec} \mathcal{O}_{X, \mathfrak{p}}}=H^{i}\left(Y_{\mathfrak{p}}, \widetilde{F_{(\mathfrak{p})}}(n)\right)^{\sim}= \begin{cases}\widetilde{\left(F_{n}\right)_{(\mathfrak{p})}} & \text { if } i=0, \\ 0 & \text { if } i>0,\end{cases}
$$

for $n>a_{X}^{*}(F)$.

To show (ii) we first observe that $\widetilde{F}(m, n)=\widetilde{F}(n) \otimes \pi^{*} \mathcal{O}_{X}(m)$. By the projection formula, we have

$$
R^{i} \pi_{*}(\widetilde{F}(m, n))=R^{i} \pi_{*}(\widetilde{F}(n)) \otimes \mathcal{O}_{X}(m)= \begin{cases}\widetilde{F_{n}}(m) & \text { if } \quad i=0 \\ 0 & \text { if } i>0\end{cases}
$$

Hence the conclusion follows from the Leray spectral sequence

$$
H^{i}\left(X, R^{j} \pi_{*}(\tilde{F}(m, n))\right) \Rightarrow H^{i+j}(Y, \tilde{F}(m, n))
$$

Let $Y$ be the blow-up of a projective scheme $X$ along an ideal sheaf $\mathcal{I}$. We say that $Y$ is locally arithmetic Cohen-Macaulay on $X$ if there exist $R$ and $I$ such that $X=\operatorname{Proj} R, \mathcal{I}=\widetilde{I}$ and $S=R[I t]$ is locally Cohen-Macaulay on $X$.

Corollary 1.4. Assume that $Y$ is locally arithmetic Cohen-Macaulay on $X$. Then

(i) $\pi_{*} \mathcal{O}_{Y}=\mathcal{O}_{X}$ and $R^{i} \pi_{*} \mathcal{O}_{Y}=0$ for $i>0$,

(ii) $H^{i}\left(Y, \mathcal{O}_{Y}(m, 0)\right) \cong H^{i}\left(X, \mathcal{O}_{X}(m)\right)$ for all $m \in \mathbb{Z}, i \geq 0$. 
Proof. With the above notations we have $a_{X}^{*}(S)=-1$ by Proposition 1.2. Hence the conclusion follows from Proposition 1.3 by taking $F=S$ and $n=0$.

For each $n$, the graded $R$-module $F_{n}$ has an $a^{*}$-invariant $a^{*}\left(F_{n}\right)$, which controls the vanishing of $H^{i}\left(X, \widetilde{F_{n}}(m)\right)$ by the Grothendieck-Serre correspondence. On the other hand, since $F$ is a finitely generated graded module over $S=R[I t]$, there exists a number $n_{0}$ such that $F_{n}=I^{n-n_{0}} F_{n_{0}}$ for $n \geq n_{0}$. It was recently discovered that for any finitely generated graded $R$-module $E$, the CastelnuovoMumford regularity $\operatorname{reg}\left(I^{n} E\right)$ is bounded by a linear function on $n$ with slope $d(I)$ [34, Theorem 2.2] (see also [10, 23] for the case $R$ is a polynomial ring). By definition, we always have

$$
a^{*}\left(I^{n} E\right) \leq \max \left\{a_{i}\left(I^{n} E\right)+i \mid i \geq 0\right\}=\operatorname{reg}\left(I^{n} E\right) .
$$

Therefore, $a^{*}\left(F_{n}\right)$ is bounded above by a linear function of the form $d(I) n+\varepsilon$ for $n \geq 1$.

We will denote by $\varepsilon(I)$ the smallest non-negative number such that

$$
a^{*}\left(I^{n}\right) \leq d(I) n+\varepsilon(I)
$$

for all $n \geq 1$. Since $\omega_{S}=\bigoplus_{n \in \mathbb{Z}} \omega_{n}$ is a finitely generated bi-graded $S$-module, there is a similar bound for $a^{*}\left(\omega_{n}\right)$. Note that the $R$-graded module $\omega_{n}$ is also called an adjoint-type module of $I$ because of its relationship to the adjoint ideals [20]. We will denote by $\varepsilon^{*}(I)$ the smallest non-negative number such that

$$
a_{i}\left(\omega_{n}\right) \leq d(I) n+\varepsilon^{*}(I)
$$

for $i \geq 2$ and $n \geq 1$.

The meaning of these invariants will become more apparent in the next sections. Here we content ourselves with the following observations.

Lemma 1.5. With the above notations we have

(i) $H^{0}\left(X, \widetilde{S_{n}}(m)\right)=S_{(m, n)}$ and $H^{i}\left(X, \widetilde{S_{n}}(m)\right)=0$ for $i>0$ and $m>d(I) n+$

(ii) $H^{i}\left(X, \widetilde{\omega_{n}}(m)\right)=0$ for $i>0$ and $m>d(I) n+\varepsilon^{*}(I)$.

Proof. Since $S_{n} \cong I^{n}$, we have $H_{R_{+}}^{i}\left(S_{n}\right)_{m}=0$ for $i \geq 0, m>d(I) n+\varepsilon(I)$ and $n \geq$ 1. Hence the first statement follows from the Serre-Grothendieck correspondence, which gives the exact sequence

$$
0 \rightarrow H_{R_{+}}^{0}\left(S_{n}\right)_{m} \rightarrow S_{(m, n)} \rightarrow H^{0}\left(X, \widetilde{S_{n}}(m)\right) \rightarrow H_{R_{+}}^{1}\left(S_{n}\right)_{m} \rightarrow 0
$$

and the isomorphisms

$$
H^{i}\left(X, \widetilde{S_{n}}(m)\right) \cong H_{R_{+}}^{i+1}\left(S_{n}\right)_{m}
$$

for $i>0$. The second statement can be similarly proved.

\section{Arithmetically Cohen-Macaulay embeddings of Blow-ups}

Let $X$ be a projective scheme over a field $k$. Let $Y \rightarrow X$ be the blowing up of $X$ along an ideal sheaf $\mathcal{I}$. We say that $Y$ has an arithmetically Cohen-Macaulay embedding if there exists a Cohen-Macaulay standard graded $k$-algebra $A$ such that $Y \cong \operatorname{Proj} A$.

Let $R$ be a finitely generated standard graded $k$-algebra and let $I \subset R$ be a homogeneous ideal such that $X=\operatorname{Proj} R$ and $\mathcal{I}$ is the ideal sheaf associated to $I$. Let $S=R[I t]$ be the Rees algebra of $R$ with respect to $I$. It is well known 
that $Y \cong \operatorname{Proj} k\left[\left(I^{e}\right)_{c}\right]$ for $c \geq d(I) e+1$ and $e \geq 1$, where $k\left[\left(I^{e}\right)_{c}\right]$ is the algebra generated by all forms of degree $c$ of the ideal power $I^{e}$ and $d(I)$ denotes the largest degree of a minimal set of homogeneous generators of $I$ (cf. [9] Lemma 1.1]). There is the following simple criterion for the existence of a Cohen-Macaulay algebra $k\left[\left(I^{e}\right)_{c}\right]$ (which is at the same time a criterion for the existence of an arithmetically Cohen-Macaulay embedding).

Lemma 2.1 ([26, Corollary 3.5]). There exists a Cohen-Macaulay ring $k\left[\left(I^{e}\right)_{c}\right]$ for $c \geq d(I) e+1$ if and only if the following conditions are satisfied:

(i) $Y$ is equidimensional and Cohen-Macaulay,

(ii) $H^{0}\left(Y, \mathcal{O}_{Y}\right)=k$ and $H^{i}\left(Y, \mathcal{O}_{Y}\right)=0$ for $i=1, \ldots, \operatorname{dim} Y-1$.

The proof of [26] used a deep result on the relationship between the local cohomology modules of a bi-graded algebra and its diagonal subalgebras 7]. However, the above lemma simply follows from the basic fact that (i) and (ii) are equivalent to the existence of an arithmetically Cohen-Macaulay Veronese embedding of $Y$ (cf. [8, Lemma 1.1]). In fact, the Veronese subalgebras of $k\left[I_{c}\right]$ are exactly the algebras of the form $k\left[\left(I^{e}\right)_{c e}\right]$ for $c \geq d(I)+1, e \geq 1$. We notice that the statements of [26. Corollary 3.5] and [8 Lemma 1.1] missed the equidimensional condition.

In this section we will determine for which values of $c$ and $e$ is $k\left[\left(I^{e}\right)_{c}\right]$ a CohenMacaulay ring. First, we show that there are well-determined invariants $\varepsilon$ and $e_{0}$ such that $k\left[\left(I^{e}\right)_{c}\right]$ is a Cohen-Macaulay ring for all $c>d(I) e+\varepsilon$ and $e>e_{0}$.

Theorem 2.2. Let $R$ be a standard graded $k$-algebra and let $I \subset R$ be a homogeneous ideal with ht $I \geq 1$. Let $Y$ be the blow-up of $X=\operatorname{Proj} R$ along the ideal sheaf of $I$ and $S=R[I t]$. Assume that

(i) $Y$ is equidimensional and Cohen-Macaulay,

(ii) $H^{0}\left(Y, \mathcal{O}_{Y}\right)=k$ and $H^{i}\left(Y, \mathcal{O}_{Y}\right)=0$ for $i=1, \ldots, \operatorname{dim} Y-1$.

Then $k\left[\left(I^{e}\right)_{c}\right]$ is a Cohen-Macaulay ring for $c>d(I) e+\max \left\{\varepsilon(I), \varepsilon^{*}(I)\right\}$ and $e>$ $\max \left\{a_{X}^{*}(S), a_{X}^{*}\left(\omega_{S}\right)\right\}$.

Note first that we always have $\max \left\{a_{X}^{*}(S), a_{X}^{*}\left(\omega_{S}\right)\right\} \geq 0$ by Proposition 1.2 and $\max \left\{\varepsilon(I), \varepsilon^{*}(I)\right\} \geq 0$ by the definition of $\varepsilon(I)$ and $\varepsilon^{*}(I)$.

Proof. Let $A=k\left[\left(I^{e}\right)_{c}\right]$. Since $c \geq d e+1$, we have $Y \cong \operatorname{Proj} A$ [9, Lemma 1.1]. On the other hand, the Rees algebra $S=R[I t]$ has a natural bi-gradation with $S_{(m, n)}=\left(I^{n}\right)_{m} t^{n}$ and $Y=\operatorname{Proj} S$. Moreover, we may view $A$ as a diagonal subalgebra of $S$; that is, $A=\bigoplus_{n \in \mathbb{N}} S_{(c n, e n)}$ [7, Lemma 1.2]. From this it follows that $A(n)^{\sim}=\mathcal{O}_{Y}(c n, e n)$. Therefore, the Serre-Grothendieck correspondence yields the exact sequence

$$
0 \longrightarrow H_{A_{+}}^{0}(A) \longrightarrow A \longrightarrow \bigoplus_{n \in \mathbb{Z}} H^{0}\left(Y, \mathcal{O}_{Y}(c n, e n)\right) \longrightarrow H_{A_{+}}^{1}(A) \longrightarrow 0
$$

and the isomorphisms

$$
\bigoplus_{n \in \mathbb{Z}} H^{i}\left(Y, \mathcal{O}_{Y}(c n, e n)\right) \cong H_{A_{+}}^{i+1}(A)
$$


for $i \geq 1$. It is well known that $A$ is a Cohen-Macaulay ring if and only if $H_{A_{+}}^{i}(A)=$ 0 for $i \neq \operatorname{dim} A$. Therefore, $A$ is a Cohen-Macaulay ring if we can show

$$
\begin{aligned}
& H^{0}\left(Y, \mathcal{O}_{Y}(c n, e n)\right)=A_{n}=\left\{\begin{array}{lll}
0 & \text { for } & n<0, \\
k & \text { for } & n=0, \\
\left(I^{e n}\right)_{c n} & \text { for } & n>0,
\end{array}\right. \\
& H^{i}\left(Y, \mathcal{O}_{Y}(c n, e n)\right)=0(i=1, \ldots, \operatorname{dim} Y-1) \text {. }
\end{aligned}
$$

For $n=0$, this follows from the assumption $H^{0}\left(Y, \mathcal{O}_{Y}\right)=k$ and $H^{i}\left(Y, \mathcal{O}_{Y}\right)=0$ for $i=1, \ldots, \operatorname{dim} Y-1$.

For $n>0$ we have $c n>d(I) e n+\varepsilon(I) n \geq d(I) e n+\varepsilon(I)$ and $e n>a_{X}^{*}(S) n \geq$ $a_{X}^{*}(S)$. Hence, using Proposition 1.3 and Lemma 1.5 we get

$$
\begin{aligned}
H^{0}\left(Y, \mathcal{O}_{Y}(c n, e n)\right) & =H^{0}\left(X, \widetilde{I^{e n}}(c n)\right)=\left(I^{e n}\right)_{c n}, \\
H^{i}\left(Y, \mathcal{O}_{Y}(c n, e n)\right) & =H^{i}\left(X, \widetilde{I^{e n}}(c n)\right)=0, i=1, \ldots, \operatorname{dim} Y-1 .
\end{aligned}
$$

For $n<0$ we have

$$
H^{i}\left(Y, \mathcal{O}_{Y}(c n, e n)\right)=H^{\operatorname{dim} Y-i}\left(Y, \omega_{Y}(-c n,-e n)\right)
$$

for $i \geq 0$. Serre duality can be applied here because $Y$ is equidimensional and Cohen-Macaulay. Since $-c n>-d(I) e n-\varepsilon^{*}(I) n \geq-d(I) e n+\varepsilon^{*}(I)$ and $-e n>$ $-a_{X}^{*}\left(\omega_{S}\right) n \geq a_{X}^{*}\left(\omega_{S}\right)$, using Proposition 1.3 and Lemma 1.5 we get

$$
H^{\operatorname{dim} Y-i}\left(Y, \omega_{Y}(-c n,-e n)\right)=H^{\operatorname{dim} Y-i}\left(X, \widetilde{\left(\widetilde{\left.\omega_{S}\right)_{-e n}}(-c n)\right)}=0\right.
$$

for $i<\operatorname{dim} Y$. So we get $H^{i}\left(Y, \mathcal{O}_{Y}(c n, e n)\right)=0$ for all $n<0$ and $i=0, \ldots, \operatorname{dim} Y-1$. The proof of Theorem 2.2 is now complete.

The following theorem shows that the bound $e>\max \left\{a_{X}^{*}(S), a_{X}^{*}\left(\omega_{S}\right)\right\}$ of Theorem 2.2 is the best possible.

Theorem 2.3. Let the notations and assumptions be as in Theorem 2.2, Let

$$
e_{0}=\max \left\{a_{X}^{*}(S), a_{X}^{*}\left(\omega_{S}\right)\right\} .
$$

Then $k\left[\left(I^{e_{0}}\right)_{c}\right]$ is not a Cohen-Macaulay ring for $c \gg 0$ if $e_{0} \geq 1$.

Proof. Let $A=k\left[\left(I^{e_{0}}\right)_{c}\right]$ for $c \gg 0$. As we have seen in the proof of Theorem 2.2 $A$ is not Cohen-Macaulay if $H^{0}\left(Y, \mathcal{O}_{Y}\left(c, e_{0}\right)\right) \neq\left(I^{e_{0}}\right)_{c}$ or $H^{i}\left(Y, \mathcal{O}_{Y}\left(c, e_{0}\right)\right) \neq 0$ or $H^{i}\left(Y, \mathcal{O}_{Y}\left(-c,-e_{0}\right)\right) \neq 0$ for some $i=1, \ldots, \operatorname{dim} Y-1$.

We shall first consider the case $e_{0}=a_{X}^{*}(S)$. Let $q$ be the smallest integer such that $e_{0}=\max \left\{a_{q}\left(S_{(\mathfrak{p})}\right) \mid \mathfrak{p} \in X\right\}$. Then

$$
\begin{aligned}
& H_{S_{(\mathfrak{p})+}}^{i}\left(S_{(\mathfrak{p})}\right)_{e_{0}}=0, i<q, \text { for all } \mathfrak{p} \in X, \\
& H_{S_{(\mathfrak{p})+}}^{q}\left(S_{(\mathfrak{p})}\right)_{e_{0}} \neq 0 \text { for some } \mathfrak{p} \in X .
\end{aligned}
$$

It is a classical result that there exists $\operatorname{dim} R_{(\mathfrak{p})}$ elements in $I_{(\mathfrak{p})}$ which generates an ideal with the same radical as $I_{(\mathfrak{p})}$. The same also holds for the ideal $S_{(\mathfrak{p})+}=$ $I_{(\mathfrak{p})} t$. From this it follows that $H_{S_{(\mathfrak{p})+}}^{\operatorname{dim} R_{(\mathfrak{p})}+1}(E)=0$ for any $R_{(\mathfrak{p})}$-module $E$ (cf. [4, Corollary 3.3.3]). Hence

$$
q \leq \max \left\{\operatorname{dim} R_{(\mathfrak{p})} \mid \mathfrak{p} \in X\right\}=\operatorname{dim} Y .
$$


Let $Y_{\mathfrak{p}}=Y \times_{X} \operatorname{Spec} \mathcal{O}_{X, \mathfrak{p}}$. The Serre-Grothendieck correspondence yields the exact sequence

$$
0 \rightarrow H_{S_{(\mathfrak{p})+}}^{0}\left(S_{(\mathfrak{p})}\right)_{e_{0}} \rightarrow\left(S_{(\mathfrak{p})}\right)_{e_{0}} \rightarrow H^{0}\left(Y_{\mathfrak{p}}, \widetilde{S_{(\mathfrak{p})}}\left(e_{0}\right)\right) \rightarrow H_{S_{(\mathfrak{p})+}}^{1}\left(S_{(\mathfrak{p})}\right)_{e_{0}} \rightarrow 0
$$

and isomorphisms $H^{i}\left(Y_{\mathfrak{p}}, \widetilde{S_{(\mathfrak{p})}}\left(e_{0}\right)\right) \cong H_{S_{(\mathfrak{p})+}^{i+1}}\left(S_{(\mathfrak{p})}\right)_{e_{0}}, i \geq 1$.

If $q \leq 1$, then $H^{0}\left(Y_{\mathfrak{p}}, \widetilde{S_{(\mathfrak{p})}}\left(e_{0}\right)\right) \neq\left(S_{(\mathfrak{p})}\right)_{e_{0}}=I_{(\mathfrak{p})}^{e_{0}}$ for some $\mathfrak{p} \in X$. From this it follows, as in the proof of Proposition 1.3 that $\pi_{*}\left(\mathcal{O}_{Y}\left(e_{0}\right)\right) \neq \widetilde{I^{e_{0}}}$. But $\pi_{*}\left(\mathcal{O}_{Y}\left(e_{0}\right)\right)(c)$ and $\widetilde{I^{e_{0}}}(c)$ are generated by global sections for $c \gg 0$. Therefore, by the projection formula we have

$$
H^{0}\left(X, \pi_{*}\left(\mathcal{O}_{Y}\left(c, e_{0}\right)\right)\right)=H^{0}\left(X, \pi_{*}\left(\mathcal{O}_{Y}\left(e_{0}\right)\right)(c)\right) \neq H^{0}\left(X, \widetilde{I^{e_{0}}}(c)\right)=\left(I^{e_{0}}\right)_{c}
$$

for $c \gg 0$. Moreover,

$$
H^{0}\left(Y, \mathcal{O}_{Y}\left(c, e_{0}\right)\right)=H^{0}\left(X, \pi_{*}\left(\mathcal{O}_{Y}\left(c, e_{0}\right)\right)\right) .
$$

Hence $H^{0}\left(Y, \mathcal{O}_{Y}\left(c, e_{0}\right)\right) \neq\left(I^{e_{0}}\right)_{c}$.

If $q \geq 2$, then the Serre-Grothendieck sequence implies $H^{i}\left(Y_{\mathfrak{p}}, \widetilde{S_{(\mathfrak{p})}}\left(e_{0}\right)\right)=0$ for all $\mathfrak{p} \in X, 0<i<q-1$, and $H^{q-1}\left(Y_{\mathfrak{p}}, \widetilde{S_{(\mathfrak{p})}}\left(e_{0}\right)\right) \neq 0$ for some $\mathfrak{p} \in X$. From this it follows, as in the proof of Proposition [1.3, that

$$
\begin{aligned}
& R^{i} \pi_{*}\left(\mathcal{O}_{Y}\left(e_{0}\right)\right)=0 \text { for } 0<i<q-1, \\
& R^{q-1} \pi_{*}\left(\mathcal{O}_{Y}\left(e_{0}\right)\right) \neq 0 .
\end{aligned}
$$

By the projection formula, we have

$$
\begin{aligned}
& R^{i} \pi_{*}\left(\mathcal{O}_{Y}\left(c, e_{0}\right)\right)=R^{i} \pi_{*}\left(\mathcal{O}_{Y}\left(e_{0}\right)\right) \otimes \mathcal{O}_{X}(c)=0 \text { for } 0<i<q-1, \\
& R^{q-1} \pi_{*}\left(\mathcal{O}_{Y}\left(c, e_{0}\right)\right)=R^{q-1} \pi_{*}\left(\mathcal{O}_{Y}\left(e_{0}\right)\right) \otimes \mathcal{O}_{X}(c) \neq 0 .
\end{aligned}
$$

Since $\pi_{*}\left(\mathcal{O}_{Y}\left(c, e_{0}\right)\right)=\pi_{*}\left(\mathcal{O}_{Y}\left(e_{0}\right)\right)(c)$, we also have $H^{q-1}\left(X, \pi_{*}\left(\mathcal{O}_{Y}\left(c, e_{0}\right)\right)\right)=0$ for $c \gg 0$. Therefore, using Leray spectral sequence

$$
H^{i}\left(X, R^{j} \pi_{*}\left(\mathcal{O}_{Y}\left(m, e_{0}\right)\right)\right) \Rightarrow H^{i+j}\left(Y, \mathcal{O}_{Y}\left(m, e_{0}\right)\right)
$$

we can deduce that

$$
H^{q-1}\left(Y, \mathcal{O}_{Y}\left(c, e_{0}\right)\right)=H^{0}\left(X, R^{q-1} \pi_{*}\left(\mathcal{O}_{Y}\left(c, e_{0}\right)\right)\right)
$$

for $c \gg 0$. But $R^{q-1} \pi_{*}\left(\mathcal{O}_{Y}\left(c, e_{0}\right)\right)$ is generated by global sections for $c \gg 0$. So we get $H^{q-1}\left(Y, \mathcal{O}_{Y}\left(c, e_{0}\right)\right) \neq 0$.

Let us now consider the case $e_{0}=a_{X}^{*}\left(\omega_{S}\right)$. Let $q$ be the smallest integer such that $e_{0}=\max \left\{a_{q}\left(\left(\omega_{S}\right)_{(\mathfrak{p})}\right) \mid \mathfrak{p} \in X\right\}$. For $\mathfrak{p} \in X$ we have $\left(\omega_{S}\right)_{(\mathfrak{p})}=\bigoplus_{n>0} H^{0}\left(Y_{\mathfrak{p}}, \omega_{Y_{\mathfrak{p}}}(n)\right)$ (see [20, 2.5.2(1) and 2.6.2]). From this it follows that $\left[H_{S_{(\mathfrak{p})+}}^{i}\left(\left(\omega_{S}\right)_{(\mathfrak{p})}\right)\right]_{n}=0$ for $n>0, i=0,1$. Since $e_{0}>0$, this implies $q>1$. Similarly as in the first case, we can also show that $q \leq \operatorname{dim} Y$ and that $H^{q-1}\left(Y, \omega_{Y}\left(c, e_{0}\right)\right) \neq 0$ for $c \gg 0$. By Serre duality we get

$$
H^{\operatorname{dim} Y-q+1}\left(Y, \mathcal{O}_{Y}\left(-c,-e_{0}\right)\right)=H^{q-1}\left(Y, \omega_{Y}\left(c, e_{0}\right)\right) \neq 0
$$

for $c \gg 0$. This completes the proof of Theorem 2.3.

We shall see later in Example 2.5 that the bound $c>d(I) e+\max \left\{\varepsilon(I), \varepsilon^{*}(I)\right\}$ of Theorem 2.2 is sharp.

Now we want to study the problem when there exists a Cohen-Macaulay ring of the form $k\left[\left(I^{e}\right)_{c}\right]$ for $e \geq 1$. 
Theorem 2.4. Let $R$ be an equidimensional standard graded $k$-algebra and let $I$ be a homogeneous ideal of $R$ with ht $I \geq 1$. Let $X=\operatorname{Proj} R$ and $S=R[I t]$. Assume that $S$ is locally Cohen-Macaulay on $X$. Then, there exists a Cohen-Macaulay ring $k\left[\left(I^{e}\right)_{c}\right]$ with $c \geq d(I) e+1$ if and only if $H^{0}\left(X, \mathcal{O}_{X}\right)=k$ and $H^{i}\left(X, \mathcal{O}_{X}\right)=0$ for $i=1, \ldots, \operatorname{dim} X-1$. In particular, this condition implies that $k\left[\left(I^{e}\right)_{c}\right]$ is a Cohen-Macaulay ring for $c>d(I) e+\max \left\{\varepsilon(I), \varepsilon^{*}(I)\right\}$ and $e \geq 1$.

Proof. Let $Y$ be the blow-up of $X$ along the ideal sheaf of $I$. The assumption implies that $Y$ is equidimensional and Cohen-Macaulay. Since $S$ is locally CohenMacaulay over $X, Y$ is locally arithmetic Cohen-Macaulay over $X$. Applying Corollary 1.4 we have $H^{0}\left(Y, \mathcal{O}_{Y}\right)=H^{0}\left(X, \mathcal{O}_{X}\right)$ and $H^{i}\left(Y, \mathcal{O}_{Y}\right)=H^{i}\left(X, \mathcal{O}_{X}\right)$ for $i>0$. Therefore, the first statement follows from Lemma 2.1. Moreover, we have $\max \left\{a_{X}^{*}(S), a_{X}^{*}\left(\omega_{S}\right)\right\}=0$ by Proposition 1.2 Hence the second statement follows from Theorem 2.2

Note that the condition $H^{0}\left(X, \mathcal{O}_{X}\right)=k$ and $H^{i}\left(X, \mathcal{O}_{X}\right)=0$ for $i=1, \ldots, \operatorname{dim} X-$ 1 is satisfied if $R$ is a Cohen-Macaulay ring.

The following example shows that the bound $c>d(I) e+\max \left\{\varepsilon(I), \varepsilon^{*}(I)\right\}$ is sharp.

Example 2.5. Let $R=k\left[x_{0}, x_{1}, x_{2}\right]$ and $I=\left(x_{1}^{4}, x_{1}^{3} x_{2}, x_{1} x_{2}^{3}, x_{2}^{4}\right)$. It is easy to see that $S=R[I t]$ is locally Cohen-Macaulay on $X=\operatorname{Proj} R$. We have $I^{n}=\left(x_{1}, x_{2}\right)^{4 n}$ for all $n \geq 2$. We have

$$
a^{*}\left(I^{n}\right)=\left\{\begin{array}{lll}
4 & \text { if } & n=1 \\
4 n-1 & \text { if } & n \geq 2 .
\end{array}\right.
$$

From this it follows that $\varepsilon(I)=0$. To compute $\varepsilon^{*}(I)$ we approximate $I$ by the ideal $J=\left(x_{1}, x_{2}\right)^{4}$. Put $S^{*}=R[J t]$. Then we have the exact sequence

$$
0 \rightarrow R[I t] \rightarrow R[J t] \rightarrow k \rightarrow 0 .
$$

From this it follows that $\omega_{S}=\omega_{S^{*}}$. Note that $S^{*}$ is a Veronese subring of the ring $T=R\left[\left(x_{1}, x_{2}\right) t\right]$ and that $T$ is a Gorenstein ring with $\omega_{T}=T(-2)$. Then $\omega_{S^{*}}=\bigoplus_{n \geq 1}\left(x_{1}, x_{2}\right)^{4 n-2}$. We have

$$
a^{*}\left(\omega_{n}\right)=a^{*}\left(\left(x_{1}, x_{2}\right)^{4 n-2}\right)=4 n-3
$$

for $n \geq 1$. Hence $\varepsilon^{*}(I)=0$. By Theorem [2.4 these facts imply that $k\left[\left(I^{e}\right)_{c}\right]$ is Cohen-Macaulay for $c>4 e$ and $e \geq 1$ (which can be also verified directly). On the other hand, for $c=4$ and $e=1$, the ring $k\left[I_{4}\right]=k\left[x_{1}^{4}, x_{1}^{3} x_{2}, x_{1} x_{2}^{3}, x_{2}^{4}\right]$ is not Cohen-Macaulay.

There have been various criteria for the Cohen-Macaulayness of Rees algebras (cf. [33, 18, 27, 30, 1, 21, 28]), so that one can construct various classes of ideals $I$ for which $S$ is locally Cohen-Macaulay on $X$. We list here only the most interesting applications of Theorem 2.4 .

Corollary 2.6. Let $R$ be a Cohen-Macaulay standard graded $k$-algebra. Let $I \subset R$ be a homogeneous ideal with ht $I \geq 1$ which is a locally complete intersection. Then $k\left[\left(I^{e}\right)_{c}\right]$ is a Cohen-Macaulay ring for all $c>d(I) e+\max \left\{\varepsilon(I), \varepsilon^{*}(I)\right\}$ and $e \geq 1$.

Proof. Let $X=\operatorname{Proj} R$. The assumption on $I$ means that $I_{\mathfrak{p}}$ is a complete intersection ideal in $R_{\mathfrak{p}}$ for $\mathfrak{p} \in X$. Therefore, $R_{(\mathfrak{p})}\left[I_{(\mathfrak{p})} t\right]$ is Cohen-Macaulay for all $\mathfrak{p} \in X$. Hence, $S=R[I t]$ is locally Cohen-Macaulay on $X$. The result follows from Theorem 2.4. 
Corollary 2.7. Let $R$ be a polynomial ring over a field $k$ of characteristic zero and $I \subset R$ a non-singular homogeneous ideal with ht $I \geq 1$. Then, $k\left[\left(I^{e}\right)_{c}\right]$ is a Cohen-Macaulay ring for $c>d(I) e+\varepsilon(I)$ and $e \geq 1$.

Proof. The assumption implies that $I$ is locally a complete intersection. Hence $S=R[I t]$ is locally Cohen-Macaulay on $X=\operatorname{Proj} R$. Let $Y=\operatorname{Proj} S$. Then $Y$ is a projective non-singular scheme. Let $m, n$ be positive integers with $m \geq$ $d(I) n+1$. Then $\mathcal{O}_{Y}(m, n)$ is a very ample invertible sheaf on $Y$ because $Y \cong$ Proj $k\left[\left(I^{n}\right)_{m}\right]$ [9 Lemma 1.1]. Let $\omega_{S}$ be the canonical module of $S$ and $\omega_{Y}=\widetilde{\omega_{S}}$. Then $H^{i}\left(Y, \omega_{Y}(m, n)\right)=0$ for $i \geq 1$ by Kodaira's vanishing theorem. On the other hand, we have

$$
H^{i}\left(Y, \omega_{Y}(m, n)\right)=H^{i}\left(X \widetilde{\left.\widetilde{\left(\omega_{S}\right)_{n}}(m)\right)}\right.
$$

by Proposition 1.3. Therefore, $H^{i}\left(X \widetilde{\left(\omega_{S}\right)_{n}}(m)\right)=0$ for $i \geq 1$. Using the SerreGrothendieck correspondence we can deduce that $H_{R_{+}}^{i}\left(\left(\omega_{S}\right)_{n}\right)_{m}=0$ for $i \geq 2$. Hence $\varepsilon^{*}(I)=0$. Now, the conclusion follows from Corollary 2.6]

Remark 2.8. A similar result to Theorem 2.4 was already given by Cutkosky and Herzog [9] Theorem 4.1] when $R$ is Cohen-Macaulay. Their result shows the existence of a constant $\delta$ such that $k\left[\left(I^{e}\right)_{c}\right]$ is Cohen-Macaulay for $c \geq \delta e, e>0$, under some assumptions on the associated graded ring $\bigoplus_{n>0} I^{n} / I^{n+1}$. It is not hard to see that these assumptions imply $\max \left\{a_{X}^{*}(S), a_{X}^{*}\left(\omega_{S}\right)\right\} \leq 0$ (see [9, Lemma 2.1 and Lemma 2.2]). Hence their result is also a consequence of Theorem 2.2 Similar statements to the above two corollaries were also given in 9 but without any information on the slope $\delta$.

It is not easy to compute $\varepsilon(I)$ explicitly, even when $I$ is a non-singular ideal in a polynomial ring. By a famous result of Bertram, Ein and Lazarsfeld [3] we know that if $I$ is the ideal of a smooth complex variety cut out scheme-theoretically by hypersurfaces of degree $d_{1} \geq \cdots \geq d_{m}$, then

$$
a_{i}\left(I^{n}\right) \leq d_{1} n+d_{2}+\cdots+d_{m}-\text { ht } I
$$

for $i \geq 2$ and $n \geq 1$. However, we do not know any bound for $a_{1}\left(I^{n}\right)$ in terms of $d_{1}, \ldots, d_{m}$. It would be of interest to find such a bound. In general, if we happen to know the minimal free resolution of $S$ over a bi-graded polynomial ring, then we can estimate $\varepsilon(I)$ in terms of the shifts of syzygy modules of the resolution [10].

In the case when $I$ is the defining ideal of a scheme of fat points, we know an explicit bound for $a^{*}\left(I^{n}\right)$, namely $a^{*}\left(I^{n}\right) \leq \operatorname{reg}(I) n$ for all $n \geq 1$ [6, 13]. As a consequence, we immediately obtain the following result of Geramita, Gimigliano and Pitteloud.

Corollary 2.9 ([13, Theorem 2.4]). Let $R$ be a polynomial ring over a field $k$ of characteristic zero, and let $I \subset R$ be the defining ideal of a scheme of fat points in Proj $R$. Then, $k\left[\left(I^{e}\right)_{c}\right]$ is a Cohen-Macaulay ring for $c>\operatorname{reg}(I) e$ and $e \geq 1$.

Proof. By definition, the ideal $I$ has the form $I=\bigcap_{i=1}^{s} \mathfrak{p}_{i}^{m_{i}}$, where $\mathfrak{p}_{i}$ is the defining prime ideal of a closed point in $X=\operatorname{Proj} R$ and $m_{i} \in \mathbb{N}$. Then $R_{(\mathfrak{p})}\left[I_{(\mathfrak{p})} t\right]$ is CohenMacaulay for all $\mathfrak{p} \in X$. In fact, we may assume that $\mathfrak{p}=\mathfrak{p}_{i}$ for some $i$. Then $\mathfrak{p}$ is a complete intersection and $R_{(\mathfrak{p})}\left[I_{(\mathfrak{p})} t\right]=R_{(\mathfrak{p})}\left[\mathfrak{p}_{(\mathfrak{p})}^{m_{i}} t\right]$ is a Veronese subalgebra of $R_{(\mathfrak{p})}\left[\mathfrak{p}_{(\mathfrak{p})} t\right]$. Since $R_{(\mathfrak{p})}\left[\mathfrak{p}_{(\mathfrak{p})} t\right]$ is a Cohen-Macaulay ring, so is $R_{(\mathfrak{p})}\left[I_{(\mathfrak{p})} t\right]$. Thus, 
$S=R[I t]$ is locally Cohen-Macaulay on $X$. This argument also shows that $Y=$ Proj $S$ is smooth. Using the Kodaira vanishing theorem we can show, as in the proof of Corollary [2.7, that $\varepsilon^{*}(I)=0$. The conclusion now follows from the proof of Theorem 2.4 when we replace the slope $d(I)$ by $\operatorname{reg}(I) \geq d(I)$ and $\varepsilon(I)$ by 0 because of the bound $a^{*}\left(I^{n}\right) \leq \operatorname{reg}(I) n$.

It was asked in 7] whether there exists a Cohen-Macaulay ring $k\left[\left(I^{e}\right)_{c}\right]$ for $c \gg$ $e \gg 0$ if $R$ is a polynomial ring and $R[I t]$ is Cohen-Macaulay. This question has been positively settled in [25. Theorem 4.5]. We can make this result more precise as follows.

Corollary 2.10. Let $R$ be a Cohen-Macaulay standard graded $k$-algebra. Let $I \subset R$ be a homogeneous ideal with ht $I \geq 1$ such that $R[I t]$ is Cohen-Macaulay. Then $k\left[\left(I^{e}\right)_{c}\right]$ is a Cohen-Macaulay ring for all $c>d(I) e+\max \left\{\varepsilon(I), \varepsilon^{*}(I)\right\}$ and $e \geq 1$.

\section{Arithmetically Cohen-Macaulay Blow-ups}

Let $X$ be a projective scheme over a field $k$. Let $\pi: Y \rightarrow X$ be the blowing up of $X$ along an ideal sheaf $\mathcal{I}$. We say that $Y$ is an arithmetically Cohen-Macaulay blow$u p$ of $X$ if there is a standard graded $k$-algebra $R$ and a homogeneous ideal $J \subset R$ with ht $J \geq 1$ such that $X=\operatorname{Proj} R, \mathcal{I}=\widetilde{J}$, and $R[J t]$ is a Cohen-Macaulay ring. The aim of this section is to characterize arithmetically Cohen-Macaulay blow-ups.

Let $R$ be a finitely generated standard graded $k$-algebra, and let $I$ be a homogeneous ideal of $R$ with ht $I \geq 1$, such that $X=\operatorname{Proj} R$ and $\mathcal{I}=\widetilde{I}$. Let $d(I)$ denote the maximal degree of the elements of a homogeneous basis of $I$. For any ideal $J$ generated by $\left(I^{e}\right)_{c}$ with $c \geq d(I) e$ we have $J_{n}=\left(I^{e}\right)_{n}$ for all $n \geq c$ so that $\mathcal{I}^{e}=\widetilde{J}$. Hence $Y=\operatorname{Proj} R[J t]$. The Rees algebra $R\left[\left(I^{e}\right)_{c} t\right]=R[J t]$ is called a truncated Rees algebra of $I^{e}$ [15] 8 . We may strengthen the problem on the characterization of arithmetically Cohen-Macaulay blow-ups by asking the question: When does there exist a Cohen-Macaulay truncated Rees algebra $R\left[\left(I^{e}\right)_{c} t\right]$ ? To solve this problem we shall need the following result of Hyry.

Let $T$ be a standard bi-graded algebra over a field $k$, that is, $T$ is generated over $k$ by the elements of degree $(1,0)$ and $(0,1)$. Let $M$ denote the maximal graded ideal of $T$ and define

$$
\begin{aligned}
& a^{1}(T):=\max \left\{m \mid \text { there is } n \text { such that } H_{M}^{\operatorname{dim} T}(T)_{(m, n)} \neq 0\right\}, \\
& a^{2}(T):=\max \left\{n \mid \text { there is } m \text { such that } H_{M}^{\operatorname{dim} T}(T)_{(m, n)} \neq 0\right\} .
\end{aligned}
$$

Theorem 3.1 ([19, Theorem 2.5]). Let $T$ be a standard bi-graded $k$-algebra with $a^{1}(T), a^{2}(T)<0$. Let $Y=\operatorname{Proj} T$. Then $T$ is Cohen-Macaulay if and only if the following conditions are satisfied:

$$
\begin{aligned}
& H^{0}\left(Y, T(m, n)^{\Upsilon}\right) \cong T_{(m, n)} \text { for } m, n \geq 0, \\
& H^{i}\left(Y, T(m, n)^{\sim}\right)=0 \text { for } m, n \geq 0, i>0, \\
& H^{i}\left(Y, T(m, n)^{\Upsilon}\right)=0 \text { for } m, n<0, i<\operatorname{dim} T-2 .
\end{aligned}
$$

Let $J \subset R$ be an arbitrary ideal generated by forms of degree $c$ and put $T=$ $R[J t]$. Then $T$ can be equipped with another bi-gradation given by

$$
T_{(m, n)}=\left(J^{n}\right)_{m+c n} t^{n}
$$

for $(m, n) \in \mathbb{N}^{2}$. With this bi-gradation, $T$ is a standard bi-graded $k$-algebra. Comparing with the natural bi-gradation of $T$ considered in the preceding sections, we 
see that both bi-gradations share the same bi-homogeneous elements and the same relevant bi-graded ideals. Therefore, Proj $T$ with respect to these bi-gradations are isomorphic.

Lemma 3.2. Let $T=R[J t]$ be as above. Then

(i) $a^{1}(T) \leq \max \left\{a^{*}\left(J^{n}\right)-n c \mid n \geq 0\right\}$,

(ii) $a^{2}(T)<0$.

Proof. To prove (i) we will show more, namely, that $H_{M}^{i}(T)_{(m, n)}=0$ for $m>$ $\max \left\{a^{*}\left(J^{n}\right)-n c \mid n \geq 0\right\}$ and $i \geq 0$. Let $T_{1}$ denote the ideal of $T$ generated by the homogeneous elements of degree $(1,0)$. Then, by [19, Lemma 2.3], we only need to show that $H_{T_{1}}^{i}(T)_{(m, n)}=0$ for $m>\max \left\{a^{*}\left(J^{n}\right)-n c \mid n \geq 0\right\}$ and $i \geq 0$. Since $T_{1}$ is generated by $R_{+}$, we always have

$$
H_{T_{1}}^{i}(T)_{(m, n)}= \begin{cases}0 & \text { for } n<0 \\ H_{R_{+}}^{i}\left(J^{n}\right)_{m+n c} & \text { for } n \geq 0 .\end{cases}
$$

But $H_{R_{+}}^{i}\left(J^{n}\right)_{m+n c}=0$ for $m+n c>a^{*}\left(J^{n}\right), n \geq 0$. Therefore, $H_{T_{1}}^{i}(T)_{(m, n)}=0$ for $m>\max \left\{a^{*}\left(J^{n}\right)-n c \mid n \geq 0\right\}$, as required.

To prove (ii) we first observe that

$$
a^{2}(T)=\max \left\{n \mid H_{M}^{\operatorname{dim} T}(T)_{n} \neq 0\right\},
$$

where the $\mathbb{Z}$-gradation comes from the natural grading $T_{n}=J^{n} t^{n}, n \geq 0$. Therefore, the conclusion $a^{2}(T)<0$ follows from [33. Corollary 3.2].

Corollary 3.3. Let $R$ be a standard graded $k$-algebra with $a^{*}(R)<0$ and let $I \subset R$ be a homogeneous ideal with ht $I \geq 1$. Let $T=R\left[\left(I^{e}\right)_{c} t\right]$ for some fixed integers $c>d(I) e+\varepsilon(I)$ and $e \geq 1$. Then $a^{1}(T)<0$ and $a^{2}(T)<0$.

Proof. Let $J$ be the ideal of $R$ generated by $\left(I^{e}\right)_{c}$. By Lemma 3.2 we only need to prove that $a^{*}\left(J^{n}\right)<n c$ for $n \geq 0$. For $n=0$, this follows from the assumption $a^{*}(R)<0$. For $n \geq 1$, we will approximate $a^{*}(J)$ by $a^{*}\left(I^{e n}\right)$. Since $J^{n}$ is generated by elements of degree $c n$ and since $c n>d(I) e n \geq d\left(I^{e n}\right)$, we have $\left(I^{e n} / J^{n}\right)_{m}=0$ for $m \geq c n$. From this it follows that $H^{0}\left(I^{e n} / J^{n}\right)=I^{e n} / J^{n}$ and $H^{i}\left(I^{e n} / J^{n}\right)=0$ for $i>0$. Therefore, from the exact sequence

$$
0 \longrightarrow J^{n} \longrightarrow I^{e n} \longrightarrow I^{e n} / J^{n} \longrightarrow 0
$$

we can deduce that $H^{i}\left(J^{n}\right)_{m}=H^{i}\left(I^{e n}\right)_{m}$ for $m \geq c n$ and $i \geq 0$. This implies

$$
a^{*}\left(J^{n}\right) \leq \max \left\{c n-1, a^{*}\left(I^{e n}\right)\right\} .
$$

By the definition of $\varepsilon(I)$ we have $a^{*}\left(I^{e n}\right) \leq d(I) e n+\varepsilon(I) \leq c n-1$. Therefore, $a^{*}\left(J^{n}\right) \leq c n-1$ for $n \geq 1$.

We are now ready to give a necessary and sufficient condition for the existence of a Cohen-Macaulay truncated Rees algebra.

Theorem 3.4. Let $R$ be a standard graded $k$-algebra with $a^{*}(R)<0$ and let $I \subset R$ be a homogeneous ideal with ht $I \geq 1$. Let $X=\operatorname{Proj} R, S=R[I t]$ and $Y=\operatorname{Proj} S$. Then there exists a Cohen-Macaulay ring $R\left[\left(I^{e}\right)_{c} t\right]$ with $c \geq d(I) e$ if and only if the following conditions are satisfied:

(i) $Y$ is equidimesional and Cohen-Macaulay,

(ii) $\pi_{*} \mathcal{O}_{Y}=\mathcal{O}_{X}$ and $R^{i} \pi_{*} \mathcal{O}_{Y}=0$ for $i>0$.

Moreover, these conditions imply that $R\left[\left(I^{e}\right)_{c} t\right]$ is a Cohen-Macaulay ring for $c>$ $d(I) e+\max \left\{\varepsilon(I), \varepsilon^{*}(I)\right\}$ and $e>\max \left\{a_{X}^{*}(S), a_{X}^{*}\left(\omega_{S}\right)\right\}$. 
Proof. Let $J$ be the ideal of $R$ generated by $\left(I^{e}\right)_{c}$ and $T=R[J t]$ for a fixed pair of positive integers $c, e$ with $c \geq d(I) e$. Then $Y \cong \operatorname{Proj} T$. If $T$ is a Cohen-Macaulay ring, then (i) is obviously satisfied and $Y$ is locally arithmetic Cohen-Macaulay over $X$. (ii) follows from Corollary 1.4

To prove the converse we equip $T$ with the aforementioned bi-gradation. Set $e_{0}=\max \left\{a_{X}^{*}(S), a_{X}^{*}\left(\omega_{S}\right)\right\}$. We will use Theorem 3.1 to prove that $T$ is CohenMacaulay for $c>d(I) e+\max \left\{\varepsilon(I), \varepsilon^{*}(I)\right\}$ and $e>e_{0}$. By Corollary 3.3 we have $a^{1}(T)<0$ and $a^{2}(T)<0$. From the bi-gradation of $T$ we see that

$$
T(m, n)^{\sim}=\mathcal{O}_{Y}(m+c n, e n),
$$

where $\mathcal{O}_{Y}(m+c n, n)$ denotes the twisted $\mathcal{O}_{Y}$-module with respect to the natural bi-gradation of $S$. If $\pi_{*} \mathcal{O}_{Y}=\mathcal{O}_{X}$ and $R^{i} \pi_{*} \mathcal{O}_{Y}=0$ for $i>0$, then we can show as in the proof of Proposition 1.3 that

$$
H^{i}\left(Y, \mathcal{O}_{Y}(m, 0)\right)=H^{i}\left(X, \mathcal{O}_{X}(m)\right)
$$

for $i \geq 0$. Since $a^{*}(R)<0$, we have $H_{R_{+}}^{i}(R)_{m}=0$ for all $m \geq 0$ and $i \geq 0$. Using the Serre-Grothendieck correspondence between sheaf cohomology of $X$ and local cohomology of $R$ we can deduce that $H^{0}\left(X, \mathcal{O}_{X}(m)\right)=R_{m}$ and $H^{i}\left(X, \mathcal{O}_{X}(m)\right)=0$ for $i>0$. Therefore,

$$
\begin{aligned}
H^{0}\left(Y, \mathcal{O}_{Y}(m, 0)\right) & =R_{m}=T_{(m, 0)} \\
H^{i}\left(Y, \mathcal{O}_{Y}(m, 0)\right) & =0, i>0
\end{aligned}
$$

For $m \geq 0$ and $n>0$ we have $m+c n>d(I) e n+\varepsilon(I)$. Therefore, using Proposition 1.3 and Lemma 1.5 we get

$$
\begin{aligned}
H^{0}\left(Y, \mathcal{O}_{Y}(m+c n, e n)\right. & =T_{(m, n)}, \\
H^{i}\left(Y, \mathcal{O}_{Y}(m+c n, e n)\right) & =0, i>0,
\end{aligned}
$$

for $e>e_{0}$. For $m, n<0$ we can show, similarly as above, that

$$
H^{i}\left(Y, \omega_{Y}(-m-c n,-e n)\right)=0
$$

for $i>0$ and $e>e_{0}$. If $Y$ is equidimensional and Cohen-Macaulay, we can apply Serre duality and obtain

$$
H^{i}\left(Y, \mathcal{O}_{Y}(m+c n, e n)\right)=0, i<\operatorname{dim} Y .
$$

Passing from $\mathcal{O}_{Y}(m+c n, e n)$ to $T(m, n)^{\sim}$ we get

$$
\begin{aligned}
& H^{0}\left(Y, T(m, n)^{\Upsilon}\right) \cong T_{(m, n)} \text { for } m, n \geq 0, \\
& H^{i}\left(Y, T(m, n)^{\Upsilon}\right)=0 \text { for } m, n \geq 0, i>0, \\
& H^{i}\left(Y, T(m, n)^{\Upsilon}\right)=0 \text { for } m, n<0, i<\operatorname{dim} T-2 .
\end{aligned}
$$

By Theorem 3.1. these conditions imply that $T$ is a Cohen-Macaulay ring. The proof of Theorem 3.4 is now complete.

The following example shows that the condition $a^{*}(R)<0$ is not necessary for the existence of a Cohen-Macaulay truncated Rees algebra. It also shows that in general, the existence of a Cohen-Macaulay truncated Rees algebra does not imply the existence of a linear bound on $c$ ensuring the Cohen-Macaulayness of $R\left[\left(I^{e}\right)_{c} t\right]$. 
Example 3.5. Take $R=k[x, y, z] /\left(x y^{2}-z^{3}\right)$, the coordinate ring of a cuspidal plane curve, and $I=(x) \subseteq R$, a homogeneous ideal with ht $I=1$. Then $R$ is a two-dimensional Cohen-Macaulay ring with $a^{*}(R)=0$. It is obvious that $R\left[\left(I^{e}\right)_{e} t\right]=R[I t]$ is a Cohen-Macaulay ring for $e \geq 1$. For $c>e$ we have $R\left[\left(I^{e}\right)_{c} t\right] \cong$ $R\left[(x, y, z)^{c-e} t\right]$. It is easy to check that the reduction number of the ideal $(x, y, z)^{c-e}$ is greater than 1. By [14], this implies that $R\left[(x, y, z)^{c-e} t\right]$ is not Cohen-Macaulay for any $c>e$.

Now we will show that the bound $e>e_{0}$ in Theorem 3.4 is once again best possible.

Theorem 3.6. Let the notations and assumptions be as in Theorem 3.4. Let

$$
e_{0}=\max \left\{a_{X}^{*}(S), a_{X}^{*}\left(\omega_{S}\right)\right\}
$$

Then $R\left[\left(I^{e_{0}}\right)_{c} t\right]$ is not a Cohen-Macaulay ring for $c \geq d(I) e_{0}$ if $e_{0} \geq 1$.

Proof. Let $T=R\left[\left(I^{e_{0}}\right)_{c} t\right]$ for some $c \geq d(I) e_{0}$, and suppose $e_{0} \geq 1$. Note that $\left(I^{e_{0}}\right)_{c}$ and $I^{e_{0}}$ defines the same ideal sheaf in $\mathcal{O}_{X}$. Consider the natural $\mathbb{N}$-grading of $T$ and $S$ given by the degree of $t$. For any $\mathfrak{p} \in X$, the $\operatorname{ring} T_{(\mathfrak{p})}$ is isomorphic to the $e_{0}$-th Veronese subring of $S_{(\mathfrak{p})}$. Hence

$$
\begin{aligned}
& H_{T_{(\mathfrak{p})+}}^{i}\left(T_{(\mathfrak{p})}\right)_{1}=H_{S_{(\mathfrak{p})+}}^{i}\left(S_{(\mathfrak{p})}\right)_{e_{0}}, \\
& H_{T_{(\mathfrak{p})+}}^{i}\left(\left(\omega_{T}\right)_{(\mathfrak{p})}\right)_{1}=H_{S_{(\mathfrak{p})+}}^{i}\left(\left(\omega_{S}\right)_{(\mathfrak{p})}\right)_{e_{0}},
\end{aligned}
$$

for $i \geq 0$. By the definition of $e_{0}$ there exists $\mathfrak{p} \in X$ and $i \geq 0$ such that either $H_{S_{(\mathfrak{p})+}}^{i}\left(S_{(\mathfrak{p})}\right)_{e_{0}} \neq 0$ or $H_{S_{(\mathfrak{p})+}}^{i}\left(\left(\omega_{S}\right)_{(\mathfrak{p})}\right)_{e_{0}} \neq 0$. Therefore, $\max \left\{a^{*}(T), a^{*}\left(\omega_{T}\right)\right\} \geq 1$. By Lemma 1.1 this implies that $T$ is not a Cohen-Macaulay ring.

From Theorem 3.4 we can derive the following sufficient condition for the existence of a truncated Cohen-Macaulay Rees algebra.

Theorem 3.7. Let $R$ be an equidimensional standard graded $k$-algebra with $a^{*}(R)<$ 0 and let $I \subset R$ be a homogeneous ideal with ht $I \geq 1$. Let $X=\operatorname{Proj} R$ and $S=R[I t]$. Assume that $S$ is locally Cohen-Macaulay on $X$. Then $R\left[\left(I^{e}\right)_{c} t\right]$ is a Cohen-Macaulay ring for $c>d(I) e+\max \left\{\varepsilon(I), \varepsilon^{*}(I)\right\}$ and $e \geq 1$.

Proof. It is obvious that the assumptions imply that $Y$ is equidimensional and Cohen-Macaulay. The condition $\pi_{*} \mathcal{O}_{Y}=\mathcal{O}_{X}$ and $R^{i} \pi_{*} \mathcal{O}_{Y}=0$ for $i>0$ follows from Corollary [1.4. Hence the conclusion follows from Theorem 3.4.

The above condition is also a necessary condition for the existence of a truncated Cohen-Macaulay Rees algebra of the form $R\left[I_{c} t\right](e=1)$.

Corollary 3.8. Let $R$ be a standard graded $k$-algebra with $a^{*}(R)<0$ and let $I \subset R$ be a homogeneous ideal with ht $I \geq 1$. Let $X=\operatorname{Proj} R$ and $S=R[I t]$. Then there exists a Cohen-Macaulay ring $R\left[I_{c} t\right]$ with $c \geq d(I)$ if and only if $S$ is locally CohenMacaulay on $X$.

Proof. By Theorem 3.7 we only need to show that if $R\left[I_{c} t\right]$ is a Cohen-Macaulay ring for some $c \geq d(I)$, then $S$ is locally Cohen-Macaulay on $X$. But this is obvious because $\left(I_{c}\right)$ and $I$ define the same ideal sheaf and $R\left[I_{c} t\right]$ is locally Cohen-Macaulay on $X$.

Using Theorem 3.7 we obtain several classes of Cohen-Macaulay Rees algebras. 
Corollary 3.9 (cf. [8, Corollary 2.2.1(2)] for the case $e=1$ ). Let $R$ be a CohenMacaulay standard graded $k$-algebra with $a(R)<0$. Let $I \subset R$ be a homogeneous ideal with ht $I \geq 1$ which is locally a complete intersection. Then $R\left[\left(I^{e}\right)_{c} t\right]$ is a Cohen-Macaulay ring for all $c>d(I) e+\max \left\{\varepsilon(I), \varepsilon^{*}(I)\right\}$ and $e \geq 1$.

Proof. As in the proof of Corollary 2.6, $S=R[I t]$ is locally Cohen-Macaulay over $X=\operatorname{Proj} R$. Since the assumption on $R$ implies $a^{*}(R)<0$, the conclusion follows from Theorem 3.7 .

Corollary 3.10. Let $R$ be a polynomial ring over a field $k$ of characteristic zero and let $I \subset R$ be a non-singular homogeneous ideal. Then $R\left[\left(I^{e}\right)_{c} t\right]$ is a CohenMacaulay ring for all $c>d(I) e+\varepsilon(I)$ and $e \geq 1$.

Proof. We have seen in the proof of Corollary 2.7 that $\varepsilon^{*}(I)=0$. Hence the assertion follows from Corollary 3.9.

Corollary 3.11 (cf. [15, Theorem 2.4] for the case $e=1$ ). Let $R$ be a polynomial ring over a field $k$ of characteristic zero and let $I \subset R$ be the defining ideal of a scheme of fat points in $\operatorname{Proj} R$. Then $R\left[\left(I^{e}\right)_{c} t\right]$ is a Cohen-Macaulay ring for $c>\operatorname{reg}(I) e$.

Proof. The proof follows from Theorem 3.7 with the same lines of arguments as in the proof of Corollary 2.9.

Now we will use Theorem 3.7 to find a criterion for arithmetically CohenMacaulay blow-ups. Recall that the blow-up $Y$ of a projective scheme $X$ along an ideal sheaf $\mathcal{I}$ is said to be locally arithmetic Cohen-Macaulay on $X$ if there exist a standard graded algebra $R$ over a field and a homogeneous ideal $I \subset R$ such that $X=\operatorname{Proj} R, \mathcal{I}=\widetilde{I}$ and $S=R[I t]$ is locally Cohen-Macaulay on $X$.

Theorem 3.12. Let $X$ be a projective scheme over a field $k$ such that $H^{0}\left(X, \mathcal{O}_{X}\right)=$ $k$ and $H^{i}\left(X, \mathcal{O}_{X}\right)=0$ for $i>0$. Let $Y \longrightarrow X$ be a proper birational morphism. Then $Y$ is an arithmetically Cohen-Macaulay blow-up if and only if $Y$ is equidimensional and locally arithmetic Cohen-Macaulay on $X$.

Proof. Suppose $Y$ is an arithmetically Cohen-Macaulay blow-up of $X$. Let $R$ be a standard graded algebra over $k$, and let $I$ be a homogeneous ideal of $R$, such that $X=\operatorname{Proj} R, Y$ is the blow-up of $X$ along the ideal sheaf $\widetilde{I}$, and $S=R[I t]$ is a Cohen-Macaulay ring. Then, $\mathcal{O}_{X, x}\left[\mathcal{I}_{x} t\right]=S_{(\mathfrak{p})}$ is obviously Cohen-Macaulay for all $\mathfrak{p} \in X$. Thus, $Y$ is locally arithmetic Cohen-Macaulay on $X$.

Conversely, suppose $Y$ is equidimensional and locally arithmetic Cohen-Macaulay on $Y$. Then there exist a standard graded $k$-algebra $R$ and a homogeneous ideal $I \subset R$ such that $X=\operatorname{Proj} R, Y$ is the blow-up of $X$ along the ideal sheaf of $I$, and $R[I t]$ is locally Cohen-Macaulay on $X$. The assumption on the sheaf cohomology of $X$ implies that $H_{R_{+}}^{i}(R)_{0}=0$ for $i \geq 0$. Without restriction we may replace $R$ by a suitable Veronese subalgebra and obtain $H_{R_{+}}^{i}(R)_{n}=0$ for all $n \geq 0$ or, equivalently, $a^{*}(R)<0$. Now we may apply Theorem 3.7 to find a Cohen-Macaulay Rees algebra $R\left[I_{c} t\right]$ with $c \gg 0$. Since the ideal $\left(I_{c}\right)$ defines the same ideal sheaf $\widetilde{I}$, we can conclude that $Y$ is an arithmetically blow-up of $X$. 


\section{NOTE ADDED IN PROOF}

After the manuscript was sent for publication, we were informed that Olga Lavila-Vidal obtained similar results in her thesis (ArXiv:math.AC/0407041) as our Theorem 2.4 and Corollary 2.6. She did not have an explicit description for the constant term as we did though.

\section{REFERENCES}

[1] I. M. Aberbach, C. Huneke and N.V. Trung. Reduction numbers, Briancon-Skoda theorems, and depth of Rees rings. Compositio Math. 97 (1995), 403-434. MR.1353282 (96g:13002)

[2] Y. Aoyama. On the depth and the projective dimension of the canonical module. Japanese J. Math. 6 (1980), 61-66. MR0615014 (82h:13007)

[3] A. Bertram, L. Ein and R. Lazarsfeld. Vanishing theorems, a theorem of Severi, and the equations defining projective varieties. J. Amer. Math. Soc. 4 (1991), no. 3, 587-602. MR 1092845 (92g:14014)

[4] M. Brodmann and R. Sharp. Local cohomology. Cambridge University Press, 1998. MR,1613627 (99h:13020)

[5] W. Bruns and J. Herzog. Cohen-Macaulay rings. Cambridge University Press, 1993. MR.1251956 (95h:13020)

[6] K. A. Chandler. Regularity of the powers of an ideal. Commun. Algebra. 25 (1997), 37733776. MR,1481564 (98i:13040)

[7] A. Conca, J. Herzog, N.V. Trung and G. Valla. Diagonal subalgebras of bi-graded algebras and embeddings of blow-ups of projective spaces. American Journal of Math. 119 (1997), 859-901. MR1465072 (99d:13001)

[8] S.D. Cutkosky and H. Tài Hà. Arithmetic Macaulayfication of projective schemes. J. Pure Appl. Algebra. To appear.

[9] S.D. Cutkosky and J. Herzog. Cohen-Macaulay coordinate rings of blowup schemes. Comment. Math. Helv. 72 (1997), 605-617. MR1600158 (99d:13028)

[10] S.D. Cutkosky, J. Herzog and N.V. Trung. Asymptotic behaviour of the CastelnuovoMumford regularity. Compositio Math. 118 (1999), 243-261. MR1711319|(2000f:13037)

[11] A.V. Geramita and A. Gimigliano. Generators for the defining ideal of certain rational surfaces. Duke Mathematical Journal. 62 (1991), no. 1, 61-83. MR1104323 (92f:14031)

[12] A.V. Geramita, A. Gimigliano and B. Harbourne. Projectively normal but superabundant embeddings of rational surfaces in projective space. J. Algebra. 169 (1994), no. 3, 791-804. MR.1302116 (96f:14044)

[13] A.V. Geramita, A. Gimigliano and Y. Pitteloud. Graded Betti numbers of some embedded rational $n$-folds. Math. Ann. 301 (1995), 363-380. MR1314592 (96f:13022)

[14] S. Goto and Y. Shimoda, On the Rees algebras of Cohen-Macaulay rings. Lect. Notes in Pure and Appl. Math. 68, Marcel-Dekker, 1979, 201-231. MR0655805 (84a:13021)

[15] H. Tài Hà. On the Rees algebra of certain codimension two perfect ideals. Manu. Math. 107 (2002), 479-501. MF1906772 (2003d:13002)

[16] H. Tài Hà. Projective embeddings of projective schemes blown up at subschemes. Math. Z. 246 (2004), 111-124. MR2031448

[17] R. Hartshorne. Algebraic Geometry. Graduate Text 52. Springer-Verlag, 1977. MR0463157 (57:3116)

[18] S. Huckaba and C. Huneke. Rees algebras of ideals having small analytic deviation. Trans. Amer. Math. Soc. 339 (1993), no. 1, 373-402. MR.1123455 (93k:13008)

[19] E. Hyry. The diagonal subring and the Cohen-Macaulay property of a multigraded ring. Trans. Amer. Math. Soc. 351 (1999), no. 6, 2213-2232. MR.1467469 (99i:13005)

[20] E. Hyry and K. Smith. On a Non-Vanishing Conjecture of Kawamata and the Core of an Ideal. Amer. J. Math. 125 (2003), 1349-1410. MR2018664

[21] B. Johnston and D. Katz. Castelnuovo regularity and graded rings associated to an ideal. Proc. Amer. Math. Soc. 123 (1995), 727-734. MR.1231300 (95d:13005)

[22] T. Kawasaki. On arithmetic Macaulayfication of local rings. Trans. Amer. Math. Soc. 354, 123-149. MR1859029 (2002i:13001)

[23] V. Kodiyalam. Asymptotic behaviour of Castelnuovo-Mumford regularity. Proc. Amer. Math. Soc. 128 (2000), 407-411. MR1621961 (2000c:13027) 
[24] D. Mumford. Varieties defined by quadratic equations. C.I.M.E. III. (1969), 29-100. MR 0282975 (44:209)

[25] O. Lavila-Vidal. On the Cohen-Macaulay property of diagonal subalgebras of the Rees algebra. Manu. Math. 95 (1998), 47-58. MR1492368 (99i:13006)

[26] O. Lavila-Vidal. On the existence of Cohen-Macaulay coordinate rings of blow-up schemes. Preprint.

[27] J. Lipman. Cohen-Macaulayness in graded algebras. Math. Res. Letters 1 (1994), 149-157. MR 1266753 (95d:13006)

[28] C. Polini and B. Ulrich. Neccessary and sufficient conditions for the Cohen-Macaulayness of blow-up algebras. Compositio Math. 119 (1999), no. 2, 185-207. MR:1723128 (2001f:13007)

[29] R. Sharp. Bass numbers in the graded case, a-invariant formula, and an analogue of Falting's annihilator theorem. J. Algebra. 222 (1999), no. 1, 246-270. MR1728160 (2000j:13027)

[30] A. Simis, B. Ulrich, and W. Vasconcelos. Cohen-Macaulay Rees algebras and degrees of plolynomial equations. Math. Ann. 301 (1995), 421-444. MF.1324518(96a:13005)

[31] I. Swanson. Powers of ideals. Primary decompositions, Artin-Rees lemma and regularity. Math. Ann. 307 (1997), 299-313. MR1428875 (97j:13005)

[32] N.V. Trung. The largest non-vanishing degree of graded local cohomology modules. J. Algebra. 215 (1999), no. 2, 481-499. MR1686202 (2000f:13038)

[33] N.V. Trung and S. Ikeda. When is the Rees algebra Cohen-Macaulay? Comm. Algebra. 17 (1989), no. 12, 2893-2922. MR1030601 (91a:13009)

[34] N.V. Trung and H-J. Wang. On the asymptotic linearity of Castelnuovo-Mumford regularity. Preprint. arXiv:math. AC/0212161.

Department of Mathematics, University of Missouri, Columbia, Missouri 65201

E-mail address: tai@math.missouri.edu

URL: http://www.math.missouri.edu/ tai/

Current address: Department of Mathematics, Tulane University, 6823 St. Charles Ave., New Orleans, Louisiana 70118

E-mail address: tai@math.tulane.edu

$U R L:$ http://www.math.tulane.edu/ tai/

Institute of Mathematics, 18 Hoang Quoc Viet, Hanoi, Vietnam

E-mail address: nvtrung@math.ac.vn 